Proceedings

\title{
RISAT-1 Compact Polarimetric Calibration and Decomposition ${ }^{+}$
}

\author{
Arun Babu ${ }^{1, *}$, Shashi Kumar ${ }^{1}$ and Shefali Agrawal ${ }^{2}$ \\ 1 Photogrammetry and Remote Sensing Department, Indian Institute of Remote Sensing, Dehradun 248001, \\ India; shashi@iirs.gov.in \\ 2 Geospatial Technology and Outreach Programme Group, Indian Institute of Remote Sensing, Dehradun \\ 248001, India; shefali_a@iirs.gov.in \\ * Correspondence: arunlekshmi1994@gmail.com; Tel.: +91-807-546-6497 \\ + Presented at the 3rd International Electronic Conference on Remote Sensing, 22 May-5 June 2018; \\ Available Online: https://sciforum.net/conference/ecrs-3.
}

Published: 23 May 2019

\begin{abstract}
Indian Space Research Organisation's Radar Imaging Satellite (RISAT) -1 was the first Synthetic Aperture Radar (SAR) satellite equipped with the compact polarimetric (CP) mode for data acquisition. To exploit the advantages offered by the $\mathrm{CP}$ mode, the datasets need to be polarimetrically calibrated. The polarimetric calibration procedure estimates the polarimetric distortions in the datasets caused due to channel imbalance, crosstalk, and Faraday rotation. These polarimetric distortions cause the misinterpretation of the ground targets in the polarimetric decomposition techniques. The Freeman compact-pol polarimetric calibration algorithm is the most commonly used algorithm. In this study, the RISAT-1 Circular Transmit Linear Receive (CTLR) dataset of the RISAT Cal Val site was used to estimate the polarimetric distortion parameters and these distortion parameters were used to polarimetrically calibrate the RISAT-1 CTLR dataset of the Doon Valley region, Uttarakhand, India. The Cloude compact-pol decomposition algorithm was used to evaluate the ground target characterization accuracy before and after polarimetric calibration using the Freeman compact-pol polarimetric calibration algorithm. Before polarimetric calibration, urban targets were showing surface scattering behavior and river channels were showing increased double-bounce scattering behavior. After polarimetric calibration, the urban targets showed dominance in double-bounce scattering and river channels showed dominance in surface scattering as per the theory.
\end{abstract}

Keywords: RISAT-1; compact polarimetry; polarimetric calibration; CTLR; polarimetric decomposition; polarimetric distortions

\section{Introduction}

In the compact polarimetry $(\mathrm{CP})$ synthetic aperture radar system architecture, only one polarization of electromagnetic wave is transmitted, which is a combination of equal weighted horizontal and vertical polarizations [1]. The CP SAR has been promoted as an alternative for the fully polarimetric SAR system because of its capability to collect similar science quality polarimetric SAR data of the ground targets with reduced hardware complexity, reduced data transmission rate, almost double swath coverage, reduced pulse repetition frequency (PRF), and with no compromise in the spatial resolution [2,3]. The most commonly developed CP SAR architecture for both spaceborne and airborne platforms is the circular transmit-linear receive (CTLR) system, which transmits the EM wave in either right or left circular polarization $(H \mp i V)$, which is achieved by transmitting both horizontal and vertical polarizations simultaneously with a phase shift of 90 degrees and receives linear horizontal $(\mathrm{H})$ and vertical $(\mathrm{V})$ polarizations simultaneously [4]. 
The polarimetric SAR data is useful in understanding the different scattering mechanisms happening on the Earth's surface from different types of targets using the phase and amplitude information from the different polarization channels data with the help of polarimetric decomposition and classification techniques [5]. However, the polarimetric distortion in the Polarimetric Synthetic Aperture Radar (PolSAR) datasets causes the polarimetric decomposition and classification techniques to produce wrong outputs, which result in misinterpreting of the scattering phenomenon and ground targets. The channel imbalance, phase bias, and crosstalk are the various types of polarimetric distortions caused due to the system non-idealities and the Faraday rotation error is the polarimetric distortion induced due to the atmosphere, which is more dominant for lowfrequency SAR systems operating in the L-band and P-band [6].

The crosstalk occurs due to the non-ideal behavior of the transmit-receive modules (TRMs) and other antenna elements. The antenna elements, which radiate the Radio Frequency (RF) signal as EM waves into the free space, are arranged in such a way that it transmits in circular polarization and receives horizontal and vertical polarization, but due to the non-ideal behavior of this antenna element, the horizontal element is also sensitive to the vertical polarization up to some extent and vice versa, leading to the crosstalk error. The channel imbalance and phase bias occurs between the different polarizations channels due to the amplifier gain mismatch between the amplifiers in the horizontal and vertical section of each TRMs. The system should transmit or receive with the same antenna gain. The channel imbalance results from the antenna gain error and it describes the amplitude or phase unconformity of the different polarization channels, both in transmission and reception [7]. Faraday rotation error occurs when an EM wave propagates through an external magnetic field. The charged ions at the ionosphere of the Earth's atmosphere causes the polarization plane of the EM wave to rotate around the radar line of sight with the Faraday rotation angle (FRA). This changes the polarization state of the EM wave, leading to Faraday rotation error. The most important error is the overestimation of volume scattering targets, like vegetation, caused due to the crosstalk and Faraday rotation errors. The band ratios using different channels results in wrong results due to the channel imbalance and phase bias between different channels [8].

Radar imaging satellite (RISAT-1) launched on April 26, 2012 by Indian Space research Organization (ISRO) was the first of its kind to build and operationalize the compact polarimetry space-borne SAR in Earth observation orbit. The uniqueness of having two separate transmit/receive modules (TRMs) to support $\mathrm{H}$ and $\mathrm{V}$ polarizations, enabled the incorporation of circular polarization in the onboard system architecture. RISAT-1 SAR, operated in the C-band (5.35 GHz), acquired the compact polarimetric data by the transmission of the EM wave in the right circular mode and coherent linear reception was done giving rise to RH and RV output channel datasets (Figure 1) [9].

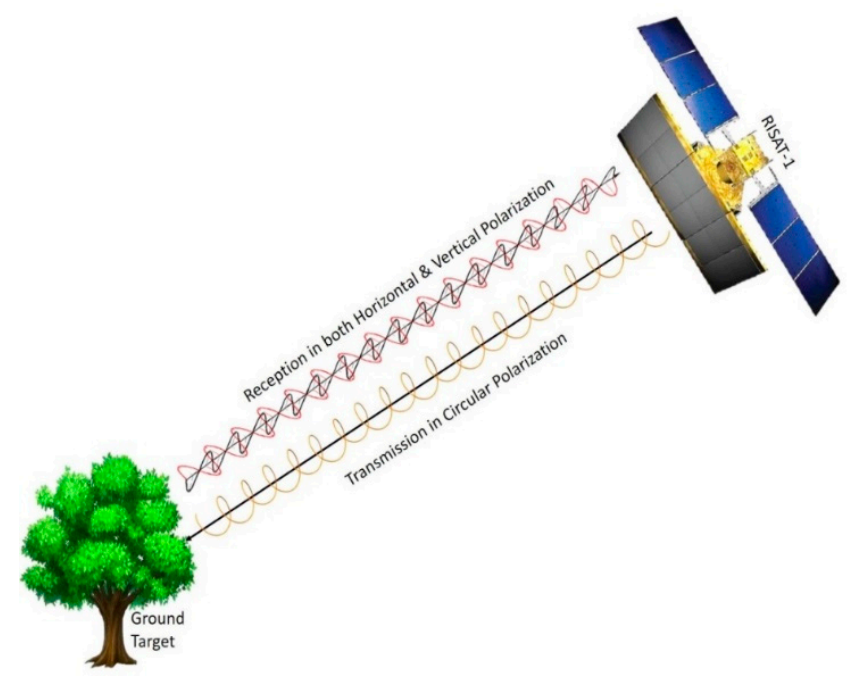

Figure 1. RISAT-1 CP-SAR data acquisition scheme. 
This study focuses on the estimation and minimization of polarimetric distortions in the RISAT1 compact-pol fine resolution Stripmap (FRS) dataset using the external polarimetric calibration techniques with the help of polarimetric distortion parameters estimated from the trihedral and dihedral corner reflectors deployed at the SAR calibration and validation site in India. The main objective of this study was to test the generalized applicability of these polarimetric distortion parameters for the polarimetric calibration of compact-pol FRS dataset of RISAT-1 acquired from anywhere on the Earth. The model-based polarimetric decomposition technique was also evaluated to assess the ground target characterization accuracy before and after polarimetric calibration.

\section{Study Area and Dataset}

\subsection{Study Area}

The Doon valley region covering Haridwar and Rishikesh, ancient cities of Uttarakhand state, in India was selected for this study. Figure 2 shows the Google Earth image of December 2014 of the Doon Valley. From the Google Earth image, it can be seen that the study area comprises of all types of possible ground targets. Water bodies, barren lands, urban structures, vegetation, and highly undulated terrain. This type of study area results in all types of possible scattering mechanisms expected from the ground targets, which is required to assess the polarimetric quality of the SAR dataset.

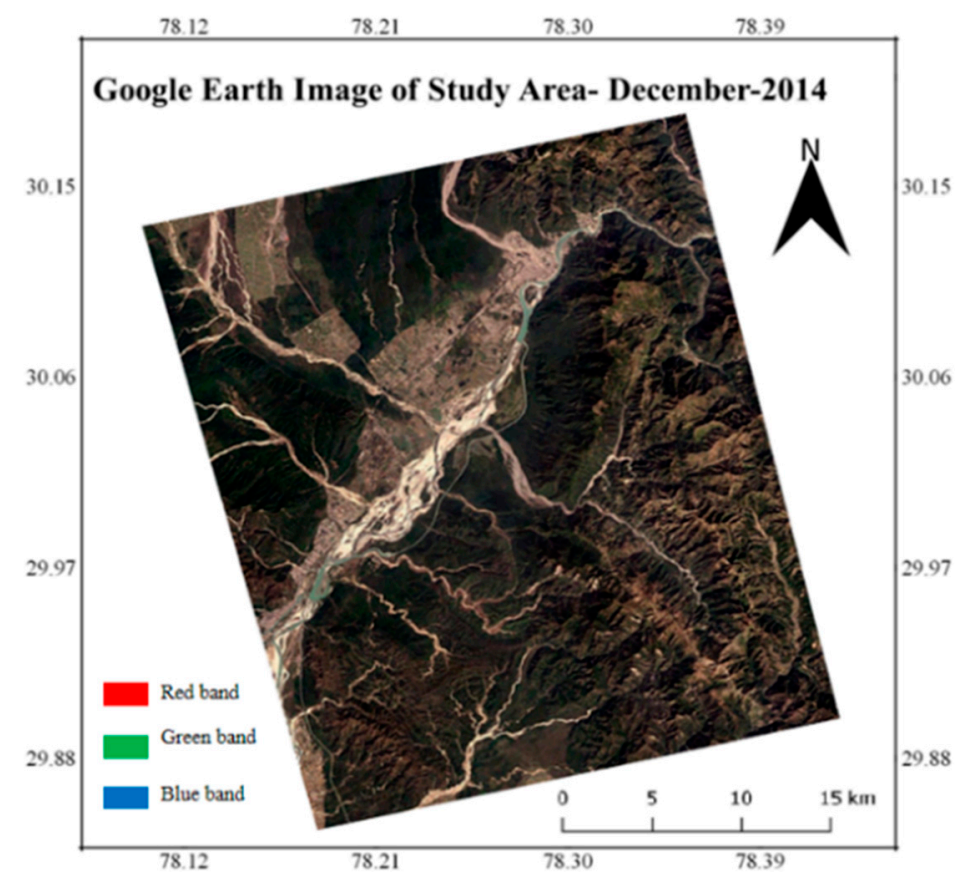

Figure 2. Study area.

\subsection{Dataset}

The RISAT-1 CP-FRS dataset of the Doon valley region acquired on November 5, 2014 was used for this study. The parameters of the datasets are given in Table 1 .

Table 1. Metadata of the dataset.

\begin{tabular}{cccccc}
\hline Date of Acquisition & Mode & Polarizations & Swath $\mathbf{( K m )}$ & Resolution $(\mathbf{A z} \times \mathbf{R g})$ & Look Direction \\
\hline 5-November 2014 & FRS-1 & RH, RV & 25 & $3 \times 2 \mathrm{~m}$ & Right \\
\hline
\end{tabular}

\section{Methodology}

The polarimetric calibration algorithm developed by Freeman et al for compact-pol data is implemented in this study [10]. In the CTLR data, since only one polarization is transmitted, it is 
impossible to correct the polarimetric distortions encountered during the signal transmission. So, in the Freeman algorithm, it is assumed that the system is perfect during signal transmission and the polarimetric distortions induced during the signal reception is estimated and corrected.

The polarimetric distortion matrix (PDM), formulated by considering the channel imbalances, crosstalk, and Faraday rotation error, is as follows [10]:

$$
\left[\begin{array}{l}
M_{R H} \\
M_{R V}
\end{array}\right]=\frac{1}{\sqrt{2}}\left[\begin{array}{cc}
1 & \delta_{2} \\
\delta_{1} & f_{1}
\end{array}\right]\left[\begin{array}{cc}
\cos \Omega & \sin \Omega \\
-\sin \Omega & \cos \Omega
\end{array}\right]\left[\begin{array}{cc}
S_{H H} & S_{H V} \\
S_{V H} & S_{V V}
\end{array}\right]\left[\begin{array}{cc}
\cos \Omega & \sin \Omega \\
-\sin \Omega & \cos \Omega
\end{array}\right]\left[\begin{array}{cc}
1 & \delta_{1} \\
\delta_{2} & f_{1}
\end{array}\right]\left[\begin{array}{c}
1 \\
-j
\end{array}\right],
$$

where $\delta_{1}$ and $\delta_{2}$ are the complex crosstalk distortion parameters during signal reception time, $f_{1}$ is the channel imbalance parameter during signal reception, and $\Omega$ is the Faraday rotation angle during signal reception. Since the signals are transmitted in the circular polarization, ideally, it will not be affected by the Faraday rotation errors. $[M]$ is the measured CTLR matrix and $[S]$ is the scattering matrix free from polarimetric distortions.

The RISAT-1 FRS-1 dataset of the Cal Val site at India where both Dihedral and Trihedral corner reflectors were deployed [11] was used to estimate the polarimetric distortion parameters using the Freeman compact-pol polarimetric calibration algorithm and the estimated polarimetric distortion parameters are shown in Table 2.

Table 2. Polarimetric distortion parameters estimated from RISAT-1 Cal Val site Fine Resolution Stripmap (FRS)-1 dataset.

\begin{tabular}{ccccc}
\hline $\begin{array}{c}\text { Distortion } \\
\text { Parameter }\end{array}$ & $\begin{array}{c}\text { Complex Crosstalk } \\
\text { Parameter ' } \boldsymbol{\delta}_{\mathbf{1}}{ }^{\prime}\end{array}$ & $\begin{array}{c}\text { Complex Crosstalk } \\
\text { Parameter ' } \boldsymbol{\delta}_{\mathbf{2}}{ }^{\prime}\end{array}$ & $\begin{array}{c}\text { Channel Imbalance } \\
\text { Parameter ' } \boldsymbol{f}_{\mathbf{1}}{ }^{\prime}\end{array}$ & $\begin{array}{c}\text { Faraday Rotation } \\
\text { Angle ' } \boldsymbol{\Omega}^{\prime}\end{array}$ \\
\hline Value & $0.8715+0.2086 \mathrm{j}$ & $-0.0770-0.1996 \mathrm{j}$ & $-0.9576+0.6808 \mathrm{j}$ & 0.01927 degrees \\
\hline
\end{tabular}

The polarimetric distortion parameter values listed in Table 2 were used to perform the polarimetric calibration of the RISAT-1 FRS-1 dataset of the Doon valley region and to estimate the polarimetrically calibrated CTLR matrix. Equation (1) was matrix inverted to perform the polarimetric calibration procedure.

The Cloude compact-pol decomposition method proposes a model-based surface/volume/dihedral technique. Initially, the covariance matrix, C2, of the compact-pol dataset is converted to the Stokes vector as follows [12]:

$$
C 2=\left[\begin{array}{ll}
\left\langle M_{R H} M_{R H}^{*}\right\rangle & \left\langle M_{R H} M_{R V}^{*}\right\rangle \\
\left\langle M_{R V} M_{R H}^{*}\right\rangle & \left\langle M_{R V} M_{R V}^{*}\right\rangle
\end{array}\right]=\frac{1}{2}\left[\begin{array}{ccc}
g_{0}+ & g_{1} & g_{2}+i g_{3} \\
g_{2}-i g_{3} & g_{0}- & g_{1}
\end{array}\right],
$$

where $[g]$ is the stokes vector. The decomposition technique relates the 4- elements of the Stokes Vector (SV) of the compact-pol dataset to the elements of the T3 matrix. This is done to correlate the stokes parameters to the scattering coefficients of the medium. Assuming refection symmetry, the T3 matrix elements are written in terms of stokes vector parameters as follows [12]:

$$
g_{s}=\left[\begin{array}{l}
g_{0} \\
g_{1} \\
g_{2} \\
g_{3}
\end{array}\right]=\left[\begin{array}{c}
\frac{1}{2}\left(t_{11}+t_{22}+t_{33}\right) \\
R e\left(t_{12}\right) \cos 2 \theta \mp \operatorname{Im}\left(t_{12}\right) \sin 2 \theta \\
-R e\left(t_{12}\right) \sin 2 \theta \mp \operatorname{Im}\left(t_{12}\right) \cos 2 \theta \\
\frac{1}{2}\left(t_{22}+t_{33}-t_{11}\right)
\end{array}\right],
$$

where $\theta$ denotes the mismatch in rotation between the scatterer symmetry axis and the radar coordinates. The model-based 3-component decomposition is implemented in Cloude compact-pol decomposition as follows [12]:

$$
\left[\begin{array}{c}
P_{D} \\
P_{V} \\
P_{S}
\end{array}\right]=\left[\begin{array}{cc}
\frac{1}{2} & g_{0} m\left(1-\cos 2 \alpha_{S}\right) \\
\frac{1}{2} & g_{0} m(1-m) \\
g_{0}\left(1+\cos 2 \alpha_{S}\right)
\end{array}\right]
$$

where $m=\frac{1}{g_{0}} \sqrt{\sum_{i=1}^{3} g_{i}^{2}}$.

The Cloude compact-pol polarimetric decomposition was implemented on both the uncalibrated and polarimetrically calibrated datasets to analyze the improvement in ground target characterization after polarimetric calibration. 


\section{Results and Discussions}

The False Color Composite (FCC) composite of the model based Cloude compact-pol decomposition technique generated from the polarimetrically uncalibrated dataset is shown in Figure 3. The red band represents the double bounce scattering component, the green band represents the volume scattering component, and the blue band represents the odd bounce scattering component. By analyzing Figure 3, it can be seen that the urban structures show surface scattering behavior instead of showing double-bounce scattering (due to the multiple scattering of the incident EM wave with the horizontal ground and vertical walls of the buildings). The dry river beds are seen in the red color because of the double bounce scattering behavior, but as per the theory, the dry river beds and other smooth surfaces should show odd bounce scattering behavior. The vegetated regions in the study area also show an overestimation of the volume scattering component because of the absence of any double-bounce scattering behavior even at the sparsely vegetated regions. Figure 4 shows the FCC composite of the model based Cloude compact-pol decomposition technique generated from the dataset after polarimetric calibration using the methodology explained in the previous section. By comparing Figures 3 and 4, it can be seen that after polarimetric calibration, there is a significant improvement in the polarimetric quality of the dataset. By analyzing the Google Earth image of the study area shown in Figure 2 and the polarimetrically calibrated FCC composite shown in Figure 4, it can be observed that the dry river beds and the water channels now appear in the blue color, indicating odd bounce scattering behavior; the urban structures now appear in red color, indicating dominance of the double bounce scattering component, and a decrease in volume scattering and increase in double-bounce scattering behavior can be observed at the less dense vegetated areas. All the scattering behaviors obtained from the ground targets now completely obey the expected scattering behaviors from these targets as per the theory.

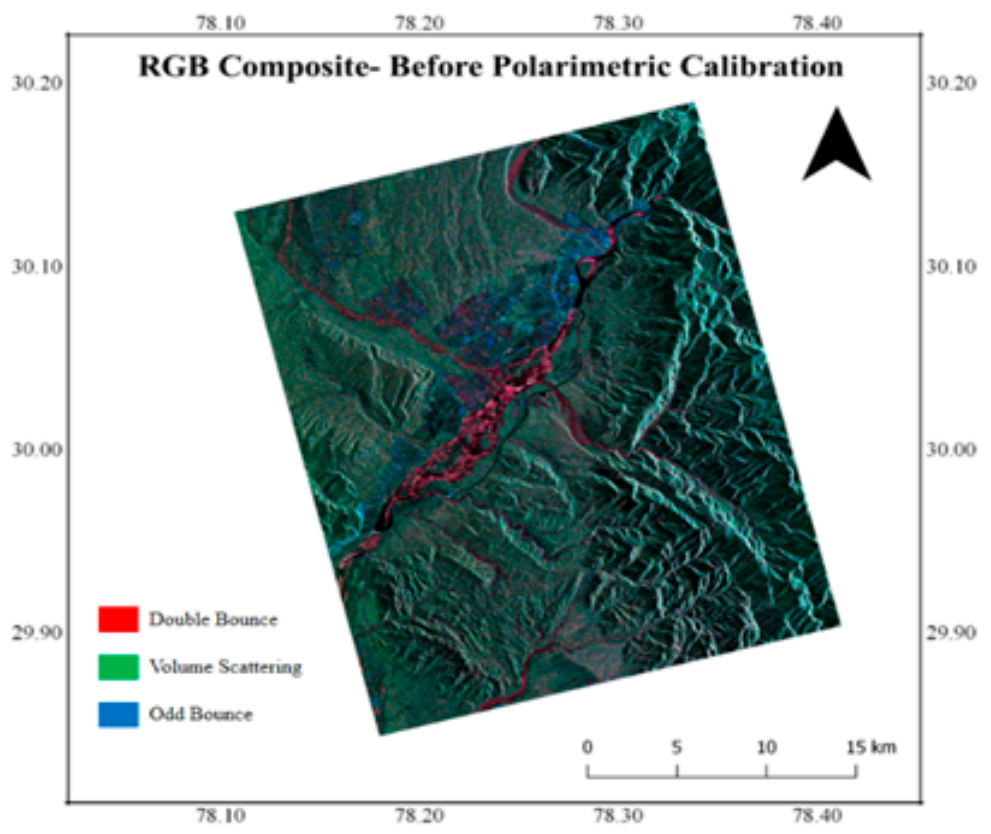

Figure 3. False Color CompositeFCC of compact-pol decomposition before polarimetric calibration. 


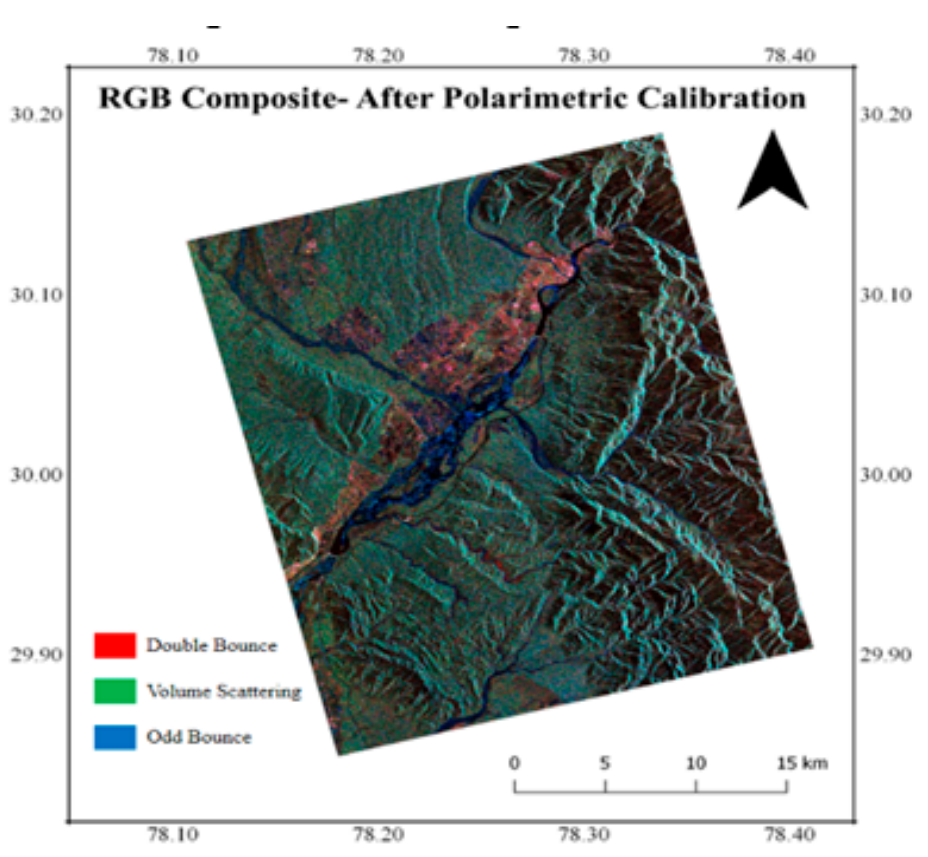

Figure 4. False Color CFCComposite of compact-pol decomposition after polarimetric calibration.

Table 3 shows the scattering magnitude values of the dry river bed, urban structures, and thick vegetation obtained from the Cloude compact-pol decomposition technique before and after polarimetric decomposition. By analyzing the table, it can be understood that the double bounce scattering and volume scattering were dominant at the dry river bed with an average magnitude of -16.09 and $-19.43 \mathrm{~dB}$, respectively, before polarimetric calibration. After polarimetric calibration, both double-bounce scattering and volume scattering magnitudes reduced to -55.46 and $-36.25 \mathrm{~dB}$, respectively, leaving behind the surface scattering component as the dominant scattering type mechanism with a magnitude of $-28.36 \mathrm{~dB}$. Before polarimetric calibration, the urban structures showed dominance in surface (odd bounce) scattering behavior with a magnitude of $-11.65 \mathrm{~dB}$ and after polarimetric calibration, the surface scattering magnitude reduced to $-49.88 \mathrm{~dB}$ and doublebounce scattering became dominant with a magnitude of $-27.36 \mathrm{~dB}$. Before polarimetric calibration, the thick vegetated regions showed high dominance in a volume scattering magnitude of $-7.73 \mathrm{~dB}$, while also showing very high surface scattering present with a magnitude of $-12.89 \mathrm{~dB}$. After polarimetric calibration, it can be observed that the volume scattering component continues as the dominant scattering mechanism with a magnitude of $-21.71 \mathrm{~dB}$ and the surface and double-bounce scattering mechanisms reduced considerably to -27.76 and $-28.40 \mathrm{~dB}$, respectively. The reduction in volume scattering magnitude at the vegetated areas indicates the reduction in the overestimation of volume scattering component after polarimetric calibration.

Table 3. Cloude compact-pol decomposition-scattering magnitude analysis.

\begin{tabular}{|c|c|c|c|c|c|c|}
\hline \multirow[b]{2}{*}{$\begin{array}{c}\text { Ground } \\
\text { Target }\end{array}$} & \multicolumn{3}{|c|}{ Before Polarimetric Calibration } & \multicolumn{3}{|c|}{ After Polarimetric Calibration } \\
\hline & $\begin{array}{l}\text { Odd Bounce } \\
\text { Scattering } \\
\text { Magnitude } \\
\text { (dB) }\end{array}$ & $\begin{array}{l}\text { Volume } \\
\text { Scattering } \\
\text { Magnitude } \\
\text { (dB) }\end{array}$ & $\begin{array}{c}\text { Double } \\
\text { Bounce } \\
\text { Scattering } \\
\text { Magnitude } \\
\text { (dB) }\end{array}$ & $\begin{array}{l}\text { Odd Bounce } \\
\text { Scattering } \\
\text { Magnitude } \\
\text { (dB) }\end{array}$ & $\begin{array}{l}\text { Volume } \\
\text { Scattering } \\
\text { Magnitude } \\
\text { (dB) }\end{array}$ & $\begin{array}{c}\text { Double } \\
\text { Bounce } \\
\text { Scattering } \\
\text { Magnitude } \\
\text { (dB) }\end{array}$ \\
\hline $\begin{array}{c}\text { Dry river } \\
\text { bed }\end{array}$ & -25.28 & -19.43 & -16.09 & -28.36 & -36.25 & -55.46 \\
\hline $\begin{array}{c}\text { Urban } \\
\text { structures }\end{array}$ & -11.65 & -15.31 & -25.93 & -49.88 & -33.52 & -27.36 \\
\hline $\begin{array}{c}\text { Thick } \\
\text { Vegetation }\end{array}$ & -12.89 & -7.73 & -21.25 & -27.76 & -21.71 & -28.40 \\
\hline
\end{tabular}




\section{Conclusions}

The compact-pol system architecture has the potential to replace the quad-pol SAR architecture because of its competing data quality and less hardware complexity. From the Cloude compact-pol decomposition results, it can be seen that the ground targets were wrongly characterized because of the misinterpretation of the scattering mechanisms caused due to the polarimetric distortions. After polarimetric calibration using the Freeman compact-pol polarimetric calibration method, it was found that the Cloude compact-pol decomposition gave accurate ground target characterization. Thus, the polarimetric calibration is a high requirement for compact-pol datasets because of the system induced and atmosphere induced polarimetric distortions. It can also be concluded that the polarimetric distortion parameters estimated from the RISAT-1 Cal Val site can be applied for any RISAT-1 FRS dataset to carry out efficient polarimetric calibration.

Acknowledgments: The authors are thankful to SAR Calibration and Validation team of Space Applications Center (SAC), ISRO, Ahmedabad for deploying the corner reflectors and providing all its details required for estimating the polarimetric distortion parameters.

Conflicts of Interest: The authors declare no conflict of interest.

\section{References}

1. Touzi, R. Compact-Hybrid versus Linear-Dual and Fully Polarimetric SAR. In Proceedings of the 4th International Workshop on Science and Applications of SAR Polarimetry and Polarimetric Interferometry, Frascati, Italy, 26-30 January 2009.

2. Jayasri, P.V. Implementation of RISAT-1 Hybrid Polarimetric Decomposition Techniques and Analysis Using Corner Reflector Data. J. Indian Soc. Remote Sens. 2018, 46, 1005-1012.

3. Raney, R.K. Hybrid-Polarity SAR Architecture. IEEE Trans. Geosci. Remote Sens. 2007, 45, 3397-3404.

4. Dubois-Fernandez, P.C.; Souyris, J.-C.; Angelliaume, S.; Garestier, F. The Compact Polarimetry Alternative for Spaceborne SAR at Low Frequency. IEEE Trans. Geosci. Rem. Sens. 2008, 376, 536-541.

5. Kumar, S.; Gupta, V.; Gonnuru, P.; Joshi, S.K. PolSAR calibration and reconstruction of hybrid polarimetric RISAT-1 data for pseudo quad-pol decomposition: a comparison with quad-pol. In Proceedings of the SPIE ASIA-PACIFIC REMOTE SENSING, New Delhi, India, 4-7 April 2016.

6. Chang, Y.; Li, P.; Yang, J.; Zhao, J.; Zhao, L.; Shi, L. Polarimetric calibration and quality assessment of the GF-3 satellite images. Sensors (Switz.) 2018, 18, 1-12.

7. Sturdivant, R.; Harris, M. Transmit/receive modules. In Transmit Receive Modules for Radar and Communication Systems; Artech House: Norwood, MA, USA, 2016; Chapter 2, Section 2.5.7; pp. 44-45.

8. Gail, W.B. Effect of Faraday rotation on polarimetric SAR. IEEE Trans. Aerosp. Electron. Syst. 1998, 34, 301308.

9. Misra, T.; Kirankumar, A.S. RISAT-1: Configuration and performance evaluation. In Proceedings of the 2014 XXXIth URSI General Assembly and Scientific Symposium (URSI GASS), Beijing, China, 16-23 August 2014; pp. 3-6.

10. Truong-Loï, M.L.; Dubois-Fernandez, P.; Pottier, E.; Freeman, A.; Souyris, J.C. Potentials of a compact polarimetric SAR system. In Proceedings of the 2010 IEEE International Geoscience and Remote Sensing Symposium, Honolulu, HI, USA, 25-30 July 2010; pp. 742-745.

11. Sharma, S.; Dadhich, G.; Rambhia, M.; Mathur, A.K.; Prajapati, R.P.; Patel, P.R.; Shukla, A. Radiometric calibration stability assessment for the risat-1 sar sensor using a deployed point target array at the desalpar site, rann of kutch, india. Int. J. Remote Sens. 2017, 38, 7242-7259.

12. Cloude, S.R.; Goodenough, D.G.; Chen, H. Compact decomposition theory. IEEE Geosci. Remote Sens. Lett. 2012, 9, 28-32.

(C) 2019 by the authors. Licensee MDPI, Basel, Switzerland. This article is an open access article distributed under the terms and conditions of the Creative Commons Attribution (CC BY) license (http://creativecommons.org/licenses/by/4.0/). 